\title{
La cultura nella funzione manageriale
}

\section{Elio Borgonovi}

Dopo la Rivoluzione industriale e soprattutto nel XX Secolo si sono affermati e sempre più consolidati il principio e la logica della specializzazione e della divisione del lavoro all'interno di istituti economici e sociali, nonché delle funzioni tra i diversi istituti nella società. In particolare si è imposta la separazione, diventata progressivamente separatezza e divaricazione, tra economia e società, tra produttività ed equità, tra produzione della ricchezza e sua distribuzione, tra scambi/relazioni di mercato e scambi/relazioni non di mercato, tra economia e cultura, tra valore economico e valore estetico, tra ricerca dell'efficienza e ricerca del bello, tra ciò che ingentilisce gli animi e ciò che risponde a bisogni materiali, di status sociale, di stima e autostima, tra approccio scientifico e approccio umanistico, tra tecnica e arte.

La presenza e l'accettazione sempre più acritica di queste dicotomie ha portato allemergere di differenti paradigmi interpretativi propri della economia e, per limitarci all'oggetto di questo contributo, della cultura. Il primo è fondato su tre pilastri: l'esplicitazione di valutazione intersoggettiva che viene oggettivata nel prezzo/valore di scambio accettato tra chi cede la proprietà e l'uso di un bene e servizio e chi lo acquisisce, la finalità di produzione e distribuzione della ricchezza economica (che è espressione del rapporto tra bisogni e limitatezza delle risorse), l'accumulazione di capitale/ patrimonio inteso come insieme condizioni idonee alla produzione futura di ricchezza. Il secondo è fondato su tre pilastri assai diversi: la presenza di un valore intrinseco della cultura sia per chi la produce sia per chi ne usufruisce che spesso esiste anche in assenza di prezzo o che comunque non viene misurato in modo adeguato dal prezzo quando esso esiste (esempio prezzo del biglietto), la produzione non direttamente o comunque non solo finalizzata ad ottenere una utilità economica, l'accumulo di capitale/ patrimonio (culturale) come insieme di condizioni idonee a suscitare percezioni di benessere intangibile nelle generazioni future.

Peraltro questa contrapposizione non sembra rispondere al mondo reale e sembra invece la conseguenza di paradigmi e di teorie "riduttivistiche", che hanno caratterizzato gli ultimi due secoli, rispetto alla multidimensionalità delle relazioni tra persone e alla poliedricità della società. Infatti, qualora si assuma una accezione ampia di cultura come insieme di valori propri e trasmessi dalle generazioni passate, conoscenze ed espressioni di persone che si traducono in concreti comportamenti, appaiono fondate le accezioni di cultura economica, cultura finanziaria, cultura di impresa, cultura del merito, cultura professionale, cultura imprenditoriale, cultura manageriale, cultura politica, cultura istituzionale e di tante altre accezioni simili tramite le quali si evidenzia che l'attività e i comportamenti umani non possono essere interpretati semplicemente con riferimento alla natura (economica e non economica) e ai contenuti delle attività svolte (economici e non 
sinergie Vol. 34, N. 99, 2016

economici). In questa prospettiva è possibile porre in evidenza alcune convergenze concettuali che si possono individuare tra attività economica di impresa e professione manageriale da un lato e il mondo della cultura dall'altro, cultura intesa come forme di espressione generate e sviluppate non per finalità dirette prevalentemente allaccumulo della ricchezza economica, come la poesia, la musica, la pittura, la danza, il teatro, e tutto ciò che in termini generali viene definito come arte. Come tutte le attività umane anche quelle di carattere artistico-culturale hanno riflessi di tipo economico, ma si vuole sottolineare che le motivazioni e le valutazioni di ordine economico non sono prioritarie come invece lo sono per la produzione di beni e servizi per il mercato che servono a soddisfare bisogni materiali o immateriali collegati allautostima o allo status sociale, al potere nella società.

Una prima convergenza riguarda la distinzione tra cultura e semplice tecnica o pratica quotidiana; infatti si parla di cultura economica, di impresa, di management per sottolineare forme di esercizio di queste funzioni che vanno oltre l'applicazione quotidiana di conoscenze: competenze, abilità e perciò sono degne di particolare riconoscibilità da parte di soggetti esterni (ad esempio colleghi di ufficio, altri manager o imprenditori, clienti e fornitori ed altri stakeholder). Allo stesso modo si parla di arte a cultura per indicare forme espressive di persone che sono degne di particolare attenzione e vanno oltre la semplice applicazione delle tecnica del disegno, della pittura, della danza, della musica, della costruzione di edifici funzionali eccetera. Perciò si parla di cultura in entrambi gli ambiti per indicare risultati di determinate attività che sono in grado di passare il filtro della rilevanza e che perciò meritano di essere osservati con particolare attenzione, assunti come termine di paragone per il progresso, trasmessi ad altri. Ne consegue che mentre la tecnica e la pratica qualificano il presente, servono per affrontare situazioni attuali, la cultura è una forma di espressione che merita di essere diffusa in una organizzazione $\mathrm{o}$ in un contesto sociale e tramandata al futuro.

La seconda convergenza deriva proprio dal riferimento temporale. Infatti la cultura nasce ed è radicata nel passato, viene interpretata e vissuta nel presente e proiettata nel futuro. Le funzioni di impresa, di imprenditori, di manager e di altre categorie economiche assurgono al rango di cultura quando esse superano la verifica del tempo. Allo stesso modo le altre espressioni umane passano dal rango di evento (musicale, teatrale, eccetera) a quello di cultura quando queste forme di espressione rimangono nella memoria di generazioni future. In questo senso va interpretata la differenza tra fare affari (o fare business come si usa dire) e sviluppare una cultura economica, cultura di impresa quest'ultima intesa come istituto economico-sociale "atto a perdurare". Allo stesso modo gli eventi teatrali, musicali, di poesia, di pittura possono essere semplice occasione di aggregazione sociale, di generazione di emozioni collettive, di business, ma essi possono diventare occasione di fruizione e generazione di cultura quando sono radicati in forme espressive del passato vivificate nel presente (fruizione) e contribuiscono a produrre cultura (significati che dal presente che si proiettano nel futuro).

Una terza convergenza che si qualifica anche come sinergia, risiede in 
diversi modi di produzione e di "fare impresa". Si fa riferimento a tutte le della funzionalità e della utilità degli oggetti quanto al collegamento tra oggetti e senso del bello, dell'armonioso, del gusto, dell'elevazione dell'animo umano. Si ricorda ad esempio la famosa Lettera 22, macchina da scrivere della Olivetti diventata oggetto di musei quali la Triennale e il MOMA, che era stata progettata su un mandato ben preciso dell'imprenditore: "costruire una macchina da scrivere che sia anche bella". I musei sia antichi che di arte moderna, contengono tanti oggetti che hanno caratterizzato le diverse "civiltà" o le diverse "epoche" (ad esempio il boom economico italiano). Venendo ad anni più recenti delleconomia si possono ricordare i prodotti della azienda Alessi che sono progettati con la logica di rappresentare anche oggetti artistici da poter mettere in mostra, quelli della Apple che ha costruito la propria immagine e il proprio successo economico sulla funzionalità dei propri computer e cellulari ma anche sulla loro bellezza e tanti altri. Un filone di ricerca che si è progressivamente affermato negli ultimi 10-15 anni tramite l'analisi di molti casi di imprese eccellenti, ha posto l'attenzione proprio sulla rilevanza competitiva del contenuto simbolico e culturale dei prodotti che rappresentano un valore aggiunto determinante rispetto a oggetti che hanno un pari, e a volte addirittura superiore, livello di funzionalità (neo-istituzionalismo dei prodotti).

Una quarta convergenza riguarda il concetto di cultura come qualcosa che le persone fanno perché hanno motivazioni intrinseche, perché credono in quello che fanno e non semplicemente in funzione dei vantaggi che ne possono trarre. Per questo alcuni imprenditori e manager si differenziano da tanti altri che svolgono le stesse funzioni, possono essere considerati in un certo senso di categoria superiore, quando diventano riferimenti "culturali" nel proprio mondo. Ciò al pari delle persone di cultura nei diversi campi che si distinguono dai semplici replicanti o da chi è solo erudito. Nella società odierna esistono imprenditori e manager, ma solo un numero limitato di essi può essere annoverato tra quelli che hanno una reale e significativa "cultura" imprenditoriale, di management e, si può aggiungere, del merito. Un numero elevato di essi si limita a cogliere "opportunità" economiche o ad applicare in modo ripetitivo e acritico metodo e tecniche di management imparati nelle università e nelle scuole di management, più o meno prestigiose. La disponibilità di tempo nei paesi progrediti, le grandi opportunità date dalla facilità di movimento, da internet e dalle nuove tecnologie, la possibilità di frequentare corsi e iniziative formative consentono a un numero elevato di persone di dedicarsi ad attività di scrittura di libri e di poesie, di pittura, di musica, di danza, di fotografia, di teatro, eccetera, ma solo un numero ristretto è riconosciuto e qualificato come persone che esprimono una cultura nel loro campo.

Una quinta convergenza riguarda l'essenza stessa della cultura che è costituita da una peculiare sintesi tra affermazione dell'io e apertura ad altri. Le persone di cultura in settori che solo per esigenze di classificazione sono definiti "economici e non economici", sono spinte da motivazioni proprie, in un certo senso da forme di sano egoismo inteso come consapevolezza di essere dotati di talenti distintivi, ma non sono interessate a tenere solo per sé i risultati. La cultura del vero imprenditore è quella di chi utilizza le 
sinergie Vol. 34, N. 99, 2016

proprie conoscenze, competenze, attitudini, capacità per generare valori anche per altri, mentre chi è molto più attento ad appropriarsi del valore generato da altri può essere definito come speculatore o opportunista. Chi è dotato di una reale "cultura" di management è orientato a governare organizzazioni moderne di maggiore o minore complessità non attraverso lesercizio del potere gerarchico secondo il modello di comando-controllo ma tramite il coinvolgimento e la valorizzazione di tutte le persone con le quali collabora a diverso livello. Le persone di cultura condividono con altri i propri saperi, le proprie intuizioni, la propria creatività e non sono interessate a tenere solo per sé le opportunità da esse generate. Proprio per questo le analisi sui paesi non sviluppati o sulle organizzazioni che decadono o addirittura muoiono mettono in evidenza non la mancanza di conoscenze e competenze di tipo imprenditoriale e manageriale, che oggi potrebbero essere facilmente trasferite, ma la mancanza o la difficoltà di consolidamento della "cultura" imprenditoriale e manageriale. Molti paesi hanno persone erudite che però non possono essere considerate colte perché non riescono a mobilitare altre persone. Le persone di cultura sono in grado di progredire e al tempo stesso di generare un "ambiente" favorevole al progresso comune allinterno delle organizzazioni e dei sistemi economici e sociali.

Una sesta convergenza, anche in questo caso fonte di sinergia, può essere ricondotta alla riscoperta da un lato e alla evoluzione dall'altro delle funzioni di imprenditori e manager. La figura dell'imprenditore da Shumpeter in poi, è stata collegata alla capacità di comprendere i bisogni che non sono ancora soddisfatti, anticipare il futuro, combinare in modo efficiente fattori produttivi scarsi, assumere il rischio, innovare in termini di prodotti, processi, mercati. La cosiddetta "creazione distruttiva" o la "distruzione creatrice" dopo la Rivoluzione industriale della fine del '700 e quella tecnologica della fine del '900 è stata collegata rispettivamente alle capacità distintive di utilizzare le conoscenze della tecnica e della tecnologia. Nell'ultimo decennio del Secolo scorso e nei primi anni del nuovo Secolo l'innovazione è sempre più collegata alla "creatività", alla riscoperta dell'interdisciplinarietà, alla capacità di coniugare la tecnica e la tecnologia ai valori umanistici. Il management che si è sviluppato nel XX Secolo soprattutto con l'applicazione dei principi del positivismo scientifico in organizzazioni complesse è stato caratterizzato da quella che alcuni autori recenti hanno definito come "capacità di fare le cose nel modo giusto", mentre oggi la domanda che ci si pone anche nel campo del management è sempre più spesso la ricerca di una risposta allesigenza di "fare nel modo giusto le cose giuste". Fare cose giuste per l'imprenditore e per il manager significa produrre beni e servizi evitando sprechi, inquinamento, rispetto dei diritti (e non solo risposte alle esigenze dei clienti), attenzione allambiente, promozione del progresso umano e sociale oltre che dello sviluppo economico. Perciò l'evoluzione delle funzioni e delle figure di imprenditori e manager è spesso sintetizzata dalle seguenti fasi: da conoscenze e competenze tecniche e professionali, a leader di organizzazioni complesse, a leader in grado di trasmettere significati profondi alle persone che fanno parte delle loro organizzazioni e a tutti coloro che con esse interagiscono. La fase finale di questa evoluzione può 
essere raggiunta solo da chi è in grado di inserire contenuti e valori umanistici sulla capacità di fare. Il connubio tra saper fare di tipo tecnico-tecnologico e dare un senso più profondo e più ampio al fare può essere favorito proprio dal collegamento tra economia, mondo dell'impresa e mondo della cultura.

In questo contributo si è cercato di sottolineare che il superamento delle dicotomie e della divaricazione del Secolo scorso e nuovi rapporti tra economia e mondo dell'impresa da un lato e mondo della cultura dall'altro vanno ben oltre le analisi che suggeriscono di collegare ai luoghi e agli eventi della cultura attività quali i bookshop, merchandising, produzione di DVD e CD-ROM, bar e ristoranti, eccetera. Vanno ben oltre lo sviluppo dei mercati dell'arte non di rado promossi e governati da "mercanti e mediatori" ben diversi dalle figure di filantropi illuminati del passato e del presente che hanno sostenuto e sostengono le espressioni nei diversi campi della cultura. Vanno oltre lo l'attenzione posta alla rilevanza dei cosiddetti settori "economici-creativi". Vanno interpretati invece con riferimento all'esigenza di ripensare i modelli economici, di impresa, di management nei quali possano essere recuperate le sinergie tra conoscenze di tipo scientifico, tecnico e tecnologico e i valori dell'umanesimo personalistico.

\section{Academic or professional position and contacts}

\section{Borgonovi Elio}

Full Professor of Business Administration and Management

University Bocconi di Milano - Italy

e-mail: elio.borgonovi@unibocconi.it 